ARTIGO

Recebido em: $30 / 10 / 2017$

Aceito em: $25 / 06 / 2018$

\title{
Comportamento de pesquisa e uso de informações irrelevantes no ambiente de trabalho
}

\author{
Search behaviour and use of irrelevant information in the work \\ environment
}

\author{
Maria Albeti Vieira VITORIANO (albeti@gmail.com)* \\ Kelley Cristine Gonçalves Dias GASQUE (kelleycristinegasque@hotmail.com) ** \\ * Doutora em Ciência da Informação pela Universidade de Brasília e Mestre em Gestão do \\ Conhecimento e da Tecnologia da Informação pela Universidade Católica de Brasília. \\ ** Doutora e Mestre em Ciência da Informação pela Universidade de Brasília, Especialista em \\ Literatura Brasileira pela Universidade Católica de Brasília. Professora Adjunta da Universidade de \\ Brasília.
}

\section{Resumo}

Revisão de literatura de natureza narrativa sobre os temas: sobrecarga de informação; informação irrelevante e pesquisa e uso de informação. Parte do pressuposto que no cotidiano os profissionais se deparam com grande quantidade de informação irrelevante para a realização das atividades profissionais. A análise da literatura mostra que o fenômeno da sobrecarga de informação é, cada vez mais, universal e onipresente na vida. 0 telefone celular (smartphone) merece papel de destaque no acesso de informações pessoais ou de lazer. As organizações conseguem limitar o acesso a informações irrelevantes somente nos dispositivos da empresa. Na maioria das vezes, os sujeitos recebem informações, eles não são ativos na busca da informação. Mais investigações sobre o tópico de pesquisa são necessárias.

Palavras-chave: Sobrecarga de informação. Informação irrelevante. Pesquisa de informação. Uso da informação.

\begin{abstract}
Literature review of narrative nature about the themes: information overload; irrelevant information; and search and use of information. It starts from the assumption that in daily life, professionals face a large amount of information that is irrelevant to the development of their professional activities. The analysis of the literature shows that the phenomenon of information overload is increasingly universal and ubiquitous in life. The smartphone has earned a prominent role in the access to personal or leisure information. Organizations can limit the access to irrelevant information only on company devices. Most of the time, the subjects receive information, they are not active in the search of information. This research topic requires further investigation.
\end{abstract}

Keywords: Information overload. Irrelevant information. Information search. Use of information.

v. 23, n. 53,2018 . p. 78-86

ISSN 1518-2924

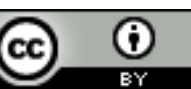

Esta obra está licenciada sob uma Licença Creative Commons 


\section{INTRODUÇÃO}

Este artigo é parte da revisão de literatura relativa à tese de Doutorado. Discorre sobre o comportamento de pesquisa e o uso de informações irrelevantes, durante o horário de trabalho. Parte-se do pressuposto de que a internet é o principal instrumento utilizado para acesso a esse tipo de informação, tanto por meio de microcomputadores como de telefones celulares (smartphones).

Nesta revisão, são abordadas questões relacionadas à sobrecarga de informação; à informação irrelevante; ao comportamento de pesquisa e uso da informação, no contexto de acesso à internet no ambiente organizacional. Trata-se de revisão de natureza narrativa (CORDEIRO et al., 2007), que apresenta temática aberta, sem pretensão de ser exaustiva. Esse tipo de revisão não requer a existência de uma questão bem definida, tampouco não exige protocolo rígido para o desenvolvimento da pesquisa. Nesse caso, a busca das fontes de informação é mais flexível e menos abrangente.

Para a supracitada revisão de literatura, foram realizadas pesquisas por meio do Portal de Periódicos da CAPES e do buscador de conteúdo Google Acadêmico, usando os seguintes descritores: "sobrecarga de informação", "informação irrelevante", "comportamento de pesquisa" e "comportamento de uso da informação", tanto na língua portuguesa, como na língua inglesa. Além disso, foram usados os descritores "cyberloafing" e "uso indevido da internet", pela semelhança de significado e, também, por não existir tradução literal daquele termo. Foram consultadas, também, algumas obras que tratam sobre esses temas.

\section{SOBRECARGA E RELEVÂNCIA DA INFORMAÇÃo}

A informação, termo por natureza polissêmico, pode ser compreendida como parte do universo físico (STONIER, 1990); ou em uma perspectiva mais complexa, como parte de uma conexão orgânica entre o universo físico, biológico e antropossociológico (MORIN, 2005). Pode, também, estar relacionada às diferentes formas de utilização: informação como processo, informação como conhecimento e informação como coisa (BUCKLAND, 1991).

Como objeto de estudo na Ciência da Informação (CI), independentemente das múltiplas perspectivas, a informação precisa estar registrada em algum tipo de suporte (LE COADIC, 2004; ROBREDO, 2007). Trata-se de um tópico que deve ser investigado, cada vez mais, pela importância que desempenha na sociedade atual. Em particular, merece especial atenção o fenômeno denominado sobrecarga de informação.

A sobrecarga de informação é causada pela grande quantidade de informação que se encontra disponível e com possibilidade de acesso pelos indivíduos. Esse volume de informação é maior do que a capacidade de absorção do indivíduo, o que demanda tempo e energia excessivos para assimilação (BAWDEN; HOLTHAM; COURTNEY, 1999; BAWDEN; ROBINSON, 2009; EPPLER; MENGIS, 2004; FARHOOMAND; DRURY, 2002).

A quantidade de informações a ser absorvida, diariamente, dificulta a capacidade de percepção e aumenta a possibilidade de compreensão equivocada das informações recebidas (WURMAN, 1989). Nesse caso, a informação recebida torna-se obstáculo, ao invés de auxiliar na execução das tarefas, podendo, também, causar descontrole sobre as atividades, sentimento de opressão e possíveis danos à saúde do indivíduo (BAWDEN; HOLTHAM; COURTNEY, 1999).

Conforme Bawden e Robinson (2009), a sensação de sobrecarga é geralmente associada a uma perda de controle sobre a situação e, às vezes, com sentimentos de opressão que, no extremo, podem produzir danos à saúde. O estado de atenção contínua e a necessidade de estar sempre conectado geram estresse, sinais de deficiência de atenção, distração e impaciência devido aos constantes estímulos mentais.

Diversos pesquisadores afirmam que a correlação positiva do desempenho de um indivíduo com a quantidade de informação que ele recebe existe, somente, até certo nível (EPPLER; MENGIS, 2004). A variedade crescente de informações pode levar à sobrecarga, tanto pelo aumento do volume de informação sobre um determinado tópico, como também pelas diferentes perspectivas apresentadas. A diversidade pode ocorrer na natureza da própria informação como no formato em que aparece. Como consequência, pode existir uma dificuldade intelectual de encaixar cada informação dentro de um quadro cognitivo apropriado (BAWDEN; ROBINSON, 2009). 
Para a Ciência Cognitiva, a atenção possui capacidade limitada, pois a memória de trabalho cria um gargalo que possibilita ao ser humano guardar na mente, somente certa quantidade de informações, num determinado instante (GOLEMAN, 2014). 0 filtro da atenção ajuda na manutenção do foco nas tarefas, de forma que só venha ocorrer alguma interrupção se a nova informação for importante, o suficiente, para interromper a concentração (LEVITIN, 2015).

0 excesso de informação, além de superar a capacidade de processamento do ser humano, cria uma conexão muito frágil de grande parte da informação acessada com os significados relevantes para o indivíduo. Assim, torna-se um desafio para o ser humano aprender a ignorar o que é irrelevante, a dar atenção ao que é importante, de forma que possa reagrupar as informações relevantes para cada contexto (LAZARTE, 2000).

Relevância é a propriedade que identifica o valor, o interesse ou a implicação da informação para o fim a que se propõe e qualifica a informação como relevante ou irrelevante (AROUCK, 2011). Informação irrelevante é aquela que não causa qualquer efeito ou facilitação para o alcance de uma meta de aprendizagem (FASS, 1994).

Nas situações em que as informações irrelevantes são detalhadas e extensas, o indivíduo pode ser conduzido a obter resultados falsos (HJELSETH, 2010). Esse tipo de informação transforma-se em ruído e em obstáculo, que devem ser erradicados, pois, tornase desinformação (SANTOS, 2005).

Saber fazer distinção entre informações relevantes e irrelevantes é um elemento fundamental para o sucesso na resolução de problemas e uma habilidade essencial (COOK, 2006). Do contrário, uma informação pode ser considerada irrelevante sem nunca ter tido sua relevância avaliada (STRAßHEIM, 2010).

Portanto, diante do fenômeno de excesso de informações, a capacidade de atenção voluntária e focada constitui fator de extrema importância para separar as informações relevantes daquelas que são irrelevantes (PIJPERS, 2010). 0 desafio dos sistemas de computador do futuro não será fornecer informação a qualquer hora e em qualquer lugar, mas dizer a coisa certa no momento certo, no caminho certo (FISCHER, 2001).

Em relação à relevância da informação, Tarapanoff (2006) argumenta que a informação irrelevante e sem precisão é pior do que nenhuma informação. Esse tipo de informação, ao invés de auxiliar o indivíduo no trabalho, cria ameaças sobre o controle das tarefas a serem realizadas, podendo causar erros, inconsistências e gerar outras informações sem valor (PIJPERS, 2010).

Kelton (2006) investigou os efeitos de informações irrelevantes sobre o julgamento de estudantes de pós-graduação, na análise financeira de uma empresa. Foram usados dois tipos de informações: informações relevantes constituídas de demonstrações financeiras auditadas; e informações irrelevantes, constantes em uma carta da administração da empresa aos acionistas. Enquanto as demonstrações financeiras mostravam um desempenho financeiro ruim, a carta da administração transmitia um tom otimista com uma perspectiva positiva para o futuro.

Após a finalização da tarefa, os participantes foram convidados a julgar a relevância de cada informação para a tomada de decisão e qual a influência no julgamento. As demonstrações financeiras foram consideradas relevantes por todos os participantes da pesquisa. Os participantes que receberam as informações relevantes e, também, a carta da administração, com informações irrelevantes, atribuíram peso, relativamente, pequeno para essas informações. No entanto, houve a percepção de que a informação irrelevante diluiu o impacto das informações relevantes, tendo afetado a tomada de decisão.

A base da pesquisa era sobre o efeito de diluição, isto é, o quanto a informação irrelevante dilui a influência da informação relevante (KELTON, 2006). O efeito de diluição ocorre quando as previsões baseadas em uma combinação de informações relevantes e não relevantes são menos consistentes do que as previsões com base apenas em informações relevantes (NISBETT; ZUKIER; LEMLEY, 1981).

$\mathrm{Na}$ visão de Kelton (2006), apesar de algumas limitações, esse estudo fornece evidências sobre os efeitos de informações irrelevantes sobre a tomada de decisão do indivíduo. Informações irrelevantes estão associadas a um aumento no esforço cognitivo necessário para processar a informação adicional e para determinar a relevância da informação.

Reconhece-se, portanto, a necessidade de identificar e selecionar as informações que são relevantes para reduzir a sobrecarga de informações. No contexto corporativo, o acesso 
às informações irrelevantes para o trabalho pode influenciar nas atividades organizacionais, como se observa no próximo tópico.

\section{PESQUISA E USO DE INFORMAÇÃO IRRELEVANTE NO AMBIENTE DE TRABALHO}

Os estudos sobre pesquisas e uso da informação estão inseridos na área de investigação denominada comportamento informacional, que se refere à totalidade do comportamento humano na busca e no uso de fontes, por meio de canais de informação (WILSON, 2000). 0 comportamento informacional abrange as diversas maneiras pelas quais o indivíduo percebe, busca, entende e usa a informação nos vários contextos da vida (CASE, 2016).

O comportamento de pesquisa de informação é considerado como o nível micro do comportamento do usuário no relacionamento com sistemas de informação de todos os tipos. Portanto, um subcampo do comportamento de busca de informação (WILSON, 1999).

Tradicionalmente, os estudos sobre pesquisa de informação estavam concentrados na busca de informação de maneira intencional, por meio da realização de pesquisas ativas. Entretanto, trabalhos mais recentes estão voltados para situações em que a informação é descoberta involuntariamente, fortuitamente e inesperadamente, muitas vezes com resultados valiosos (ERDELEZ; MAKRI, 2011). Bates (2002) e Savolainen (2016) representam essa tendência de integração dos estudos sobre busca e pesquisa de informação, principalmente aquelas realizadas por meio da internet no cotidiano do ser humano.

Bates (2002) procura integrar a busca e pesquisa de informação, utilizando duas variáveis: i) ativa versus passiva e ii) direcionada versus não direcionada. A primeira variável indica se o indivíduo faz alguma ação, de forma ativa, para buscar informação ou se mantém uma postura passiva, apenas disponível para absorver a informação, que não está procurando. A segunda variável refere-se ao indivíduo que procura informações específicas ou, apenas, encontra-se exposto, de forma aleatória, às informações disponíveis.

Com base nessa proposição, Savolainen (2016) apresenta uma estrutura conceitual com quatro modos típicos de busca de informação (Figura 1).

\begin{tabular}{l|l|l|}
\multicolumn{1}{c}{ Ativo } & \multicolumn{2}{c|}{ Passivo } \\
\cline { 2 - 3 } Direcionada & a) Busca e pesquisa ativa & $\begin{array}{l}\text { b) Monitoração } \\
\text { passiva }\end{array}$ \\
\cline { 2 - 3 } N ão direcionada & $\begin{array}{l}\text { c) Navegação e } \\
\text { visualização }\end{array}$ & $\begin{array}{l}\text { d) Acquisição incidental } \\
\text { de informação }\end{array}$ \\
\hline
\end{tabular}

Figura 1 - Modos de busca de informação

Fonte: Savolainen (2016)

A busca e a pesquisa ativa de informação (célula a), bem como a monitoração passiva (célula b) são formas de encontrar informações para atender necessidades que são reconhecidas e que ainda não foram satisfeitas. Navegação e visualização (célula c) e aquisição incidental de informação (célula d) são formas não direcionadas de encontrar informação, das quais o indivíduo toma consciência, somente, quando acessa algo que lhe desperta interesse.

O comportamento de uso da informação, outra subárea de estudo do comportamento informacional humano, refere-se à incorporação de informações na base de conhecimento existente em cada indivíduo. Do ponto de vista da psicologia evolutiva, o uso da informação é visto como o processo pelo qual os dados coletados são usados para 
transformar a perspectiva do indivíduo e permitir a adaptação do comportamento às necessidades do ambiente (SPINK; COLE, 2006).

0 uso da informação altera o estado de conhecimento do indivíduo ou a capacidade de ação. Trata-se de um processo relacionado à seleção e ao processamento de informação, que pode responder a uma pergunta, solucionar um problema, auxiliar na tomada de decisão ou possibilitar o entendimento de uma situação (CHOO, 2003).

De forma complementar, Choo (2003) argumenta que o uso efetivo da informação está relacionado ao caráter de relevância cognitiva e emocional conferido pelo indivíduo à informação recebida, assim como aos atributos objetivos que determinam a pertinência da informação quanto à resolução de determinada situação problema.

A busca de informação, portanto, é um aspecto essencial do comportamento integral do ser humano. Buscar informações, constantemente, é vital para viver, adaptar-se às transformações da sociedade, tomar decisões e resolver problemas, tanto na vida pessoal como no aspecto profissional. A evolução da tecnologia da informação e a expansão da internet têm possibilitado e estimulado cada vez mais essa característica do ser humano.

Nesse cenário, as organizações mudaram a forma de conduzir suas atividades. Muitos benefícios, tais como: agilidade na comunicação; melhoria no acesso e na distribuição de informações; redução de custos e do prazo de entrega de produtos e serviços são resultados da adoção de novas tecnologias, principalmente, aquelas disponibilizadas pela internet (HENLE; BLANCHARD, 2008; SAWITRI, 2012).

Em relação aos empregados, as tecnologias atuais possibilitam o aumento da produtividade, o controle das atividades e o crescimento de oportunidades de desenvolvimento profissional. Em contrapartida, essa configuração favorece o uso da internet, para fins pessoais, com inúmeros reflexos negativos para as organizações, principalmente, prejuízo nas atividades profissionais (FEZILE, 2011; SAWITRI, 2012),

De acordo com Gonçalves (2017), os recursos de tecnologia da informação e da comunicação alteram o modus operandi dos indivíduos. Além dos microcomputadores, os telefones celulares são, cada vez mais, usados para acessar a internet, em casa, na rua, na escola, no clube e no trabalho. Nas organizações, esse comportamento pode causar prejuízos no desempenho das atividades profissionais e até mesmo criar problemas relacionados à segurança da informação corporativa (KHANSA et al., 2017).

0 termo cyberloafing é utilizado para designar o envolvimento de profissionais, de forma regular, em atividades não relacionadas ao trabalho por meio do acesso à internet (LIM, 2002). Essas atividades podem ser, por exemplo, navegar na internet, participar de jogos on-line ou, simplesmente, acessar emails pessoais, durante o horário de trabalho (HENLE; BLANCHARD, 2008; KHANSA et al., 2017). Outros autores usam as expressões uso pessoal da web (personal web usage - PWU), abuso do uso da internet, computação não relacionada ao trabalho e uso problemático de internet para referenciar esse tipo de comportamento (KWON, 2015; LEE, Y.; LEE, Z.; KIM, 2007).

Khansa et al. (2017) relatam que alguns mecanismos são utilizados com o objetivo de controlar essa situação, como políticas de uso da internet e adoção de mecanismos de bloqueio do acesso a alguns tipos de site, ao correio eletrônico pessoal e às redes sociais. No entanto, Richards (2011) considera que esse controle é uma tarefa difícil pelo potencial das tecnologias de comunicação da internet, que permitem o acesso sem precedentes e, potencialmente, ilimitado ao mundo fora do ambiente de trabalho, no horário de expediente e as expensas da organização.

No entanto, ao mesmo tempo em que esse tipo de atividade - cyberloafing - é considerado como um desvio da atividade profissional, também, pode ser visto como uma forma de ajudar a quebrar a monotonia do trabalho e reduzir o estresse devido à carga excessiva de trabalho. Além disso, medidas de contenção do uso da internet tem causado descontentamento entre os profissionais, com repercussão no comportamento laboral, inclusive, possíveis atitudes de retaliação, afirmam KHANSA et al. (2017).

Pesquisa realizada no Reino Unido, por um escritório de advocacia trabalhista, demonstra que trabalhadores ingleses gastam $40 \%$ do tempo navegando na internet, em sites não relacionados às atividades profissionais e/ou acessando o correio eletrônico pessoal. O levantamento envolveu 3.245 trabalhadores, de 750 empresas, do quais $97 \%$ admitiram usar a internet para uso pessoal em média três horas por dia (MANAGEMENT ISSUES NEWS, 2004). 
Examinando por outro ângulo, pesquisa realizada pela American Management Association mostra que $47 \%$ dos empregadores verificam o conteúdo do email e $63 \%$ monitoram o uso da internet dos empregados. Além disso, mais de $50 \%$ já haviam empregado alguma sanção coercitiva ou demitido pelo menos um empregado pelo uso indevido do correio eletrônico ou da internet e 38\% utilizam software que impede o acesso a sites de internet inapropriados (LARA, 2006).

Estudo realizado por Kwon (2015), com 198 pessoas, da Universidade de Otago (Nova Zelândia) encontrou os seguintes resultados: 42\% dos respondentes acessaram alguma rede social entre uma a quatro vezes por dia; $52 \%$ nunca acessaram redes sociais e, uma minoria, $1 \%$ fez algum tipo de pesquisa mais do que 20 vezes por dia. Nessa pesquisa, foi percebida diferença no uso da internet para fins pessoais entre a equipe acadêmica e os demais profissionais.

Em relação ao Brasil, Gonçalves (2017) apresenta uma pesquisa realizada com gestores organizacionais públicos e privados, num total de 212 respondentes. Alguns resultados merecem ser destacados, como por exemplo, 53,6 \% dos respondentes admitem que os empregados priorizam a comunicação pessoal (facebook, whatsapp, email e outros) em detrimento do trabalho.

No entanto, somente, 29,8\% afirmam que existe preocupação nas organizações com o uso crescente de dispositivos pessoais de tecnologia da comunicação, durante o horário de trabalho. Além disso, somente, 36,4 \% admitem que os resultados da organização são prejudicados pelo uso pessoal, abusivo e indiscriminado das tecnologias da comunicação. E finalmente, 76,8 \% concordam que medidas de restrição ao uso da internet nas organizações desestabilizam os profissionais.

De acordo com Gonçalves (2017), essa pesquisa sobre o fenômeno que ele denomina como dependência digital é inédita no Brasil, principalmente, em relação às organizações. Verifica-se, portanto, que ainda existe um campo bastante vasto para ser investigado no que se refere ao uso da internet no trabalho para fins pessoais. Tanto no que se refere ao tempo despendido nessa atividade, como em relação à relevância, ou não, das informações acessadas, como também, em relação às medidas adotadas pelas organizações e os impactos no comportamento dos profissionais.

\section{CONSIDERAÇÕES FINAIS}

Esta revisão não pretende esgotar o universo de publicações existentes sobre a pesquisa e o uso de informações irrelevantes, no ambiente de trabalho, por meio da internet. Trata-se de um tema de considerável abrangência, com possibilidade de severas implicações para o trabalho nas organizações. As referências utilizadas, na sua maioria, são publicações na língua inglesa; no Brasil, somente uma pesquisa sobre esse tema foi localizada.

A título de conclusão, alguns pontos merecem ser destacados. O fenômeno da sobrecarga de informação é, cada vez mais, universal e onipresente no cotidiano do ser humano, tanto no aspecto pessoal, como nas atividades profissionais. Nesse cenário, merece destaque o papel do telefone celular (smartphone) que possibilita o acesso a diversos conteúdos, redes sociais e inúmeros aplicativos, muitos dos quais sem qualquer relevância para a vida e para as atividades dos indivíduos.

No que se refere ao ambiente de trabalho, as organizações procuram restringir o acesso à internet, por meio do bloqueio de acesso a alguns sítios, redes sociais e correio eletrônico. No entanto, essas medidas estão limitadas ao microcomputador, não abrangem o celular, que pode ser usado pelo profissional para acessar as informações disponíveis na internet que, em geral, são irrelevantes para as atividades profissionais.

Em relação à busca de informação, as pesquisas parecem estar mais restritas ao formato de navegação e visualização, resultando na aquisição incidental de informação. Ou seja, os indivíduos estão, na maioria das vezes, sendo receptores de informações e não sujeitos ativos na busca do que lhes parece ser relevante para a execução de tarefas e tomadas de decisão na vida e no trabalho.

Dessa forma, acredita-se que existe um campo imenso a ser investigado, nos mais diversas áreas do comportamento informacional humano, tanto no aspecto pessoal como em relação às atividades e ao desempenho profissional. A rede mundial de computadores e, consequentemente, a grande quantidade de informações disponíveis, são fatos presentes na 
sociedade atual. Trazem grandes benefícios para a humanidade, porém, geram grandes desafios e problemas que precisam ser investigados e enfrentados. Nesse contexto, a CI pode desempenhar importante papel no desenvolvimento de estudos interdisciplinares ao lado de outras áreas do conhecimento.

\section{REFERÊNCIAS}

AROUCK, Osmar. Atributos de qualidade da informação. 2011. Dissertação (Mestrado em Ciência da Informação). Programa de Pós-Graduação em Ciência da Informação, Faculdade de Ciência da Informação, Universidade de Brasília, Brasília, 2011.

BATES, Marcia J. Toward an integrated model of information seeking and searching. New Review of Information Behaviour Research, v. 3, p. 1-15, 2002. Disponível em: $<$ https://pages.gseis.ucla.edu/faculty/bates/articles/info SeekSearch-i-030329.html $>$. Acesso em: 04 out. 2017.

BAWDEN, David; HOLTHAM, Clive; COURTNEY, Nigel. Perspectives on information overload. Aslib Proceedings, v. 51, n. 8, p. 249-255, set. 1999. Disponível em: <http://www.emeraldinsight.com/doi/abs/10.1108/EUM0000000006984?journalCode=ap $>$. Acesso em: 04 out. 2017.

BAWDEN, David; ROBINSON, Lyn. The dark side of information: overload, anxiety and other paradoxes and pathologies. Journal of Information Science, v. 35, n. 2, p. 180-191, 2009. Disponível em: $<$ http://www.city.ac.uk/ data/assets/pdf file/0020/79103/IIS-dark-side.pdf >. Acesso em: 01 out. 2017.

BUCKLAND, Michael. Information as Thing. Journal of the American Society of Information Science, v. 42, n. 5, p. 351-360, jun. 1991. Disponível em: <http://skat.ihmc.us/rid=1KR7VC4CQ-SLX5RG5T39/BUCKLAND(1991)-informationasthing.pdf >. Acesso em: 30 set. 2017.

CASE, Donald O. Looking for Information: a survey of research on information seeking, needs, and behavior. 4. ed. Edição do Kindle. United Kingdom: Emerald, 2016.

CHOO, Chun W. A Organização do Conhecimento: como as organizações usam a informação para criar significado, construir conhecimento e tomar decisões. São Paulo: SENAC, 2003.

CORDEIRO, Alexander Magno et al. Revisão sistemática: uma revisão narrativa. Revista do Colégio Brasileiro de Cirurgiões, Rio de Janeiro, v. 34, n. 6, p. 428-431, nov./dez. 2007. Disponível em: <http://www.scielo.br/pdf/rcbc/v34n6/11.pdf>. Acesso em: 07 set. 2017.

COOK, Joan Littlefield. College students and algebra story problems: strategies for identifying relevant information. Reading Psychology, v.27, p. 95-125, 2006. Disponível em: <http://www.tandfonline.com/doi/abs/10.1080/02702710600640198?journalCode=urpy20>. Acesso em: 29 set. 2017.

EPPLER, Martin J.; MENGIS, Jeanne. The Concept of Information Overload: a review of literature from organization science, accounting, Marketing, MIS, and Related Disciplines. The information society: an international journal, v. 20, n. 5, p. 1-20, 2004. Disponível em: https://link.springer.com/chapter/10.1007\%2F978-3-8349-9772-2 15. Acesso em: 28 set. 2017.

ERDELEZ, Sanda; MAKRI, Stephan. Introduction to the thematic issue on opportunistic discovery of information. Information Research, v. 16, n. 3, set. 2011.

FARHOOMAND, Ali F.; DRURY, Don H. Managerial information overload. Communications of the ACM, v. 45, n. 10, out. 2002. Disponível em: $\leq$ http://dl.acm.org/citation.cfm?doid=570907.570909>. Acesso em: 27 abr. 2017.

FASS, Leona F. Learning From Relevant and Irrelevant Information. In: GREINER, Russ; SUBRAMANIAN, Devika (Eds.) AAAI Technical Report FS-94-02. Menlo Park: AAAI Press, 1994. Disponível em: $<$ http://www.aaai.org/Papers/Symposia/Fall/1994/FS-94-02/FS94-02-019.pdf $>$. Acesso em: 27 set. 2017.

FEZILE, Ozdamli. Are teacher candidates problematic Internet users? Procedia - Social and Behavioral Sciences, v. 30, p. 2345-2349, 2011. Disponível em: < https://www.sciencedirect.com/science/article/pii/S1877042811022828>. Acesso em: 26 out. 2017.

FISCHER, Gerhard. Articulating the Task at Hand and Making Information Relevant to It. Humancomputer interaction, v.16, p. 243-256, 2001. Disponível em: <http://13d.cs.colorado.edu/ gerhard/papers/hci2001.pdf >. Acesso em: 25 set. 2017.

GOLEMAN, Daniel. Foco: a atenção e seu papel fundamental para o sucesso. Rio de Janeiro: Objetiva, 2014 
GONÇALVES, Lucio L. Dependência Digital: tecnologias transformando pessoas, relacionamentos e organizações. Rio de Janeiro: Barra Livros, 2017.

HENLE, Christine A.; BLANCHARD, Anita L. The interaction of work stressors and organizational sanctions on cyberloafing. Journal of Managerial Issues, v. 20, n. 3, p. 383-400, 2008. Disponível em: <https://www.questia.com/library/journal/1G1-188064188/the-interaction-of-work-stressors-andorganizational>. Acesso em: 26 out. 2017.

HJELSETH, Eilif. Exchange of relevant information in BIM objects defined by the role- and life-cycle information model. Architectural engineering and design management, v. 6, p. 279-287, 2010. Disponível em: <http://www.tandfonline.com/doi/abs/10.3763/aedm.2010.IDDS5?src=recsys $>$. Acesso em: 29 set. 2017.

KELTON, Andrea S. Internet financial reporting: the effects of hyperlinks and irrelevant information on investor judgments. 2006. 42f. Dissertação (Master in Accounting and Information Management). Department of Accounting and Information Management, University of Tennessee, Knoxville, 2006. Disponível em: $\leq$ https://mgt.ncsu.edu/pdfs/accounting/kelton_dissertation_1-19-06.pdf > . Acesso em: 25 set. 2017.

KHANSA, Lara; BARKHI, Reza; RAY, Soumya; DAVI, Zachary. Cyberloafing in the workplace: mitigation tactics and their impact on individuals' behavior. Information Technology and Management, set. 2017. Disponível em: <https://link.springer.com/article/10.1007/s10799-017-0280-1>. Acesso em: 04 out. 2017.

KWON, Kean F. Implications of internet usage policy on personal web usage from the perspective of senior management and other staff members. 2015. Tese (Master in Science) University of Otago, Dunedin, New Zealand. 2015. Disponível em:

<https://ourarchive.otago.ac.nz/bitstream/handle/10523/5821/KwonKF2015MSc.pdf;sequence=1>. Acesso em: 04 out. 2017.

LARA, Pablo Z. Manrique de. Fear in organizations: does intimidation by formal punishment mediate the relationship between interactional justice and workplace internet deviance? Journal of Managerial Psychology, v. 21, n. 6, p. 580-592, 2006. Disponível em: <http://www.emeraldinsight.com/toc/imp/21/6>. Acesso em: 25 set. 2017.

LAZARTE, Leonardo. Ecologia cognitiva na sociedade da informação. Ciência da Informação, v. 29, n. 2, p. 43-51, mai./ago. 2000. Disponível em: <http://www.scielo.br/pdf/\%0D/ci/v29n2/a06v29n2.pdf>. Acesso em: 26 set. 2015.

LE COADIC, Yves-Francois. A ciência da informação. Brasília: Briquet de Lemos, 2004.

LEE, Younghwa; LEE, Zoonky; KIM, Yongbeom. Understanding personal web usage in organizations. Journal of Organizational Computing and Electronic Commerce, v. 17, n. 1, 2007. Disponível em:

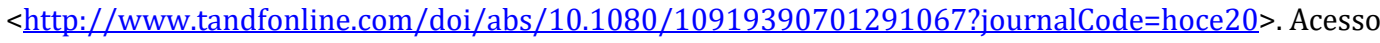
em: 26 out. 2017.

LEVITIN, Daniel J. A mente organizada: como pensar com clareza na era da sobrecarga de informação. Rio de Janeiro: Objetiva, 2015.

LIM,Vivien K. G. The IT way of loafing on the job: cyberloafing, neutralizing and organizational justice. Journal of Organizational Behavior, v. 23, n 5, p. 675- 694, aug. 2002. Disponível em: <http://onlinelibrary.wiley.com/doi/10.1002/job.161/abstract>. Acesso em: 26 out. 2017.

MANAGEMENT ISSUES NEWS. Does cyberloafing undermining productivity? 2004. Disponível em: $<$ http://www.management-issues.com/news/1417/does-cyberloafing-undermining-productivity/>. Acesso em: 24 set. 2017.

MORIN, Edgar. 0 método: a natureza da natureza. Porto Alegre: Sulina, 2005.

NISBETT, Richard. E.; ZUKIER, Henry; LEMLEY, Ronald E. The dilution effect: nondiagnostic information weakens the implications of diagnostic information. Cognitive Psychology, v. 13, p. 248277, 1981. Disponível em: <http://www.sciencedirect.com/science/article/pii/0010028581900104>. Acesso em: 25 set. 2017.

PIJPERS, Guus. Information Overload: a system for better managing everyday data. Nova Jersei: Wiley, 2010.

RICHARDS, James. What has the internet ever done for employees? A review, map and research agenda. Employee Relations, v. 34, n.1, p. 22 - 43, 2011. Disponível em:

<http://www.emeraldinsight.com/doi/abs/10.1108/01425451211183246>. Acesso em: 04 out. 2017. 
ROBREDO, Jaime. Filosofia da ciência da informação ou Ciência da informação e filosofia? In: TOUTAIN, Lídia M. B. Brandão (Org.). Para entender a ciência da informação. Salvador: EDUFBA, 2007.

SANTOS, B. de Sousa. Os tribunais e as novas tecnologias de comunicação e de informação. Sociologias, Porto Alegre, ano 7, n. 13, jan./jun. 2005, p. 82-109. Disponível em:

$<$ http://seer.ufrgs.br/sociologias/article/view/550>. Acesso em: 23 out. 2017.

SAWITRI, Hunik S.R. Role of internet experience in moderating influence of work stressor on cyberloafing. Procedia - Social and Behavioral Sciences, v. 57, out. 2012, p. 320-324. Disponível em: <http://www.sciencedirect.com/science/article/pii/S187704281204654X?via\%3Dihub>. Acesso em: 26 out. 2017.

SAVOLAINEN, Reijo. Elaborating the conceptual space of information-seeking phenomena. Information research, v. 21, n. 3, set. 2016. Disponível em: <http://www.informationr.net/ir/213/paper720.html\#.WP4C8dLyvIU>. Acesso em: 24 set. 2017.

SPINK, Amanda; COLE, Charles. Human information behavior: integrating diverse approaches and information use. Journal of the American Society for Information Science and Technology, v.57, n.1, p.25-35, 2006. Disponível em: <http://onlinelibrary.wiley.com/doi/10.1002/asi.20249/abstract>. Acesso em: 22 set. 2017.

STONIER, T. Information and the internal structure of universe: an exploration into information physics. New York: Springer -Verlag, 1990.

STRAßHEIM, Jan. Relevance theories of communication Alfred Schutz in dialogue with Sperber and Wilson. Journal of Pragmatics, v. 42, p. 1412-1441, 2010. Disponível em: $<$ http://www.academia.edu/1314867/Relevance_theories_of_communication_Alfred_Schutz_in_dialog ue with Sperber and Wilson>. Acesso em: 22 set. 2017.

TARAPANOFF, Kira. Informação, conhecimento e inteligência em corporações: relações e complementaridade. In: TARAPANOFF, Kira (org.), Inteligência, informação e conhecimento. Brasília: IBICT, 2006.

WILSON, T. D. Human information behavior. Informing Science Research. v.3, n.2, 2000. Disponível em: $\leq$ https://pdfs.semanticscholar.org/f393/bbfdac03c71f567887513c0e2687df8cd412.pdf $>$. Acesso em: 30 set. 2017.

WILSON, T. D. Models in information behavior research. Journal of Documentation, London, v.55, n.3, p.249-270, jun. 1999. Disponível em: <http://www-emeraldinsightcom.ez54.periodicos.capes.gov.br/doi/pdfplus/10.1108/EUM0000000007145>. Acesso em: 24 set. 2017.

WURMAN, R. Saul. Ansiedade de Inform@ção: como transformar informação em compreensão. São Paulo: Cultura, 1989.

Editores do artigo: Enrique Muriel-Torrado, Edgar Bisset Alvarez, Camila Barros. 